\title{
Fecal Microbiota Transplantation for Ulcerative Colitis: FoMenTing Change?
}

\author{
Flaviano S. Martins ${ }^{1}$
}

Published online: 22 March 2016

(C) Springer Science+Business Media New York 2016

Fecal microbiota transplant (FMT), the process of introducing fecal bacteria from a healthy donor into a diseased patient, has gained increasing attention since it was established as an effective treatment for patients suffering from infection with Clostridium difficile (CD), the pathogenic bacterium responsible for pseudomembranous colitis and for some antibiotic-associated diarrhea. Due to the mounting failure experienced with eliminating this bacterium with conventional therapies, alternative treatments have been used, of which the most promising is FMT [1]. The concept of using feces to treat gastrointestinal diseases was first documented in China in the fourth century and in the USA in 1958 [2, 3]. In 2013, a randomized controlled trial published in The New England Journal of Medicine concluded that FMT is significantly more effective for the treatment of recurrent CD infection (CDI) than the use of antibiotics [4].

Since FMT has captured the attention of the public, it is being tested for a number of diseases other than CDI, including ulcerative colitis (UC), which along with Crohn's disease is one of the two major forms of a condition known as inflammatory bowel diseases (IBD), a group of chronic idiopathic inflammatory conditions that affect the gastrointestinal tract. Although the pathogenesis of IBD is still not fully understood, an inappropriate inflammatory response against intestinal microbiota in genetically susceptible individuals appears to be a key component of disease pathogenesis [5]. The treatment of IBD consists of

Flaviano S. Martins

flaviano@icb.ufmg.br

1 Laboratory of Biotherapeutic Agents, Department of Microbiology, Institute of Biological Sciences, Federal University of Minas Gerais, Belo Horizonte, Brazil immunomodulatory therapy that at times is supplemented by surgical interventions aiming to ensure longer remission periods and to prevent possible complications. Since dysbiosis, defined as an alteration of gut microbial species thought to be pathologic, is thought to contribute to IBD pathogenesis [6], the use of probiotics or FMT has been proposed as alternative therapies [7]. Although the efficacy of FMT has yet to be unambiguously supported in UC on the basis of very few randomized trials [8], the use of animal models to better understand the mechanism underlying this new approach is now being explored.

In this issue of Digestive Diseases and Sciences, Tian and colleagues report the novel observation that FMT is beneficial in a well-established mouse model of chemically induced colitis termed the dextran sodium sulfate (DSS) model [9]. Improved clinical and histological scores were observed in animals that received FMT after the colitis induction, with little evidence of an inflammatory infiltrate in the intestines after FMT was stopped.

Using metagenomic analysis, the authors observed that colitis is associated with a relative preponderance of the bacterial genera Bacteroides and Lactobacillus and the species Escherichia coli, whereas the population of Bifidobacterium (a known fermentative bacterium) was decreased. Except for Bacteroides, FMT altered the distributions of the three genera and one species toward the ratios observed in controls. The observation, derived from the study of gem-free animals, that bacteria are required for the initiation of intestinal inflammation of IBD, complemented by the finding that dysbiosis accompanies gut inflammation, supports the use of FMT as an alternative treatment for UC. In the context of the inflammatory response observed in IBD, Tian and colleagues also observed that the alteration of regulatory lymphocytes and cytokine expression associated with colitis induction in 
mice was reversed after FMT, with the inflammatory pattern similar to that of healthy control mice (naïve group). The DSS-induced colitis mouse model, which is associated with increased gut paracellular permeability, in many ways resembles clinical disease.

With regard to the use of probiotics or FMT to treat UC in a murine model $[7,10,11]$, FMT is a more efficient therapy [9], perhaps related to the quantity and quality of bacteria, since FMT involves the entire fecal microbiota, whereas probiotic treatment introduces only a single or a few bacterial strains. The microbiota of healthy donors may act as a "broad-spectrum antibiotic" against pathogens with an added benefit of adding needed bacterial species, normalizing the overall microbial composition. Nevertheless, the World Health Organization (WHO) recommends the administration of live microbial cells only if the microorganisms are thoroughly defined in terms of taxonomic identity, with no known virulence factors. Although application of these constraints is possible for probiotics, it is not possible for FMT, since fecal samples of apparently healthy donor could present some potentially pathogenic microorganisms or viral particles at residual levels. The process of selection of the ideal healthy donor is a key step in FMT, due to the considerable variation of microbial composition among individuals.

The use of FMT, particularly in animal models, is subject to some caveats, since there is considerable variation of microbial composition among donors, which, combined with the genetic variations among the recipients, can directly influence the success of the colonization and fecal transplant therapy. By using two different mouse strains (BALB/c and C57BL/6 J mice), the authors observed that FMT did not improve the disease activity index nor prevent the death of C57BL/6 J mice, suggesting that the genetic background influences experimental outcomes. In the author's words: "Different genetic predisposition and treatment responses to both the disease and the alteration of microbiota may complicate clinical application of FMT to UC patients. Individualized rational therapeutic approach should be based on one's genome sequence data and unique gut microbiota." Furthermore, although the DSS model is associated with increased mucosal paracellular permeability and dysbiosis, it does not model the genetic origins related to IBD, which in addition to the donor microbiota quality can introduce multiple variables given the 133 IBD identified disease-associated genes [9].

Other questions that should be addressed include the ideal frequency of FMT needed for long-term effectiveness; the risks of the introduction of exogenous gut bacteria into an inflamed, leaky colon; the possibility that donor microorganisms can be "rejected" in the usual sense; and effectively identifying potential pathogens hidden in the donor's fecal microbiota.

Although little mechanistic knowledge exists about this new approach, FMT as first-line therapy unquestionably helps ameliorate CDI and is a promising adjuvant therapy for IBD patients. Since preclinical studies must precede clinical testing of a new therapy, the work of Tian and colleagues is a first step toward treating UC with alternative therapies such as FMT.

\section{References}

1. Brown WR. Fecal microbiota transplantation in treating Clostridium difficile infection. J Dig Dis. 2014;15:405-408.

2. Zhang F, Luo W, Shi Y, Fan Z, Ji G. Should we standardize the 1,700-year-old fecal microbiota transplantation? Am J Gastroenterol. 2012;107:1755.

3. Eiseman B, Silen W, Bascon GS, Kauvar AJ. Fecal enema as an adjunct in the treatment of pseudomembranous enterocolitis. Surgery. 1958;44:854-859.

4. van Nood E, Vrieze A, Nieuwdorp M, et al. Duodenal infusion of donor feces for recurrent Clostridium difficile. $N$ Engl J Med. 2013;368:407-415.

5. Matricon J, Barnich N, Ardid D. Immunopathogenesis of inflammatory bowel disease. Self Nonself. 2010;1:299-309.

6. Ghouri YA, Richards DM, Rahimi EF, et al. Systematic review of randomized controlled trials of probiotics, prebiotics, and synbiotics in inflammatory bowel disease. Clin Exp Gastroenterol. 2014;7:473-487.

7. Souza ÉL, Elian SD, Paula LM, et al. Escherichia coli strain Nissle 1917 ameliorates experimental colitis by modulating intestinal permeability, inflammatory response and clinical signs in a faecal transplantation model. J Med Microbiol. 2016. doi:10. 1099/jmm.0.000222.

8. Moayyedi P, Surette MG, Kim PT, et al. Fecal microbiota transplantation induces remission in patients with active ulcerative colitis in a randomized controlled trial. Gastroenterology. 2015;149:102-109.

9. Tian Z, Liu J, Liao M, et al. Beneficial effects of fecal microbiota transplantation on ulcerative colitis in mice. Dig Dis Sci. (Epub ahead of print). doi:10.1007/s10620-016-4060-2.

10. Elian SD, Souza EL, Vieira AT, et al. Bifidobacterium longum subsp. infantis BB-02 attenuates acute murine experimental model of inflammatory bowel disease. Benef Microbes. 2015;6:277-286.

11. Tiago FC, Porto BA, Ribeiro NS, et al. Effect of Saccharomyces cerevisiae strain UFMG A-905 in experimental model of inflammatory bowel disease. Benef Microbes. 2015;6:807-815. 